\title{
Type 4 dual left anterior descending coronary artery
}

\author{
Chan Joon Kim, Hee Jeoung Yoon, Sung Ho Her, Jun Han Jeon, Seung Min Jung, Eun Hee Jang, \\ and Seung Won Jin
}

Division of Cardiology, Department of Internal Medicine, College of Medicine, Daejeon St. Mary's Hospital, The Catholic University of Korea, Daejeon, Korea
Received: March 4, 2008

Revised : March 25, 2008

Accepted: September 8, 2008

\section{Correspondence to}

Hee Jeoung Yoon, M.D.

Division of Cardiology,

Department of Internal Medicine,

College of Medicine, Daejeon St.

Mary's Hospital, The Catholic

University of Korea, 64

Daeheung-ro, Jung-gu, Daejeon

34943 , Korea

Tel: +82-42-220-9505

Fax: +82-42-253-9505

E-mail: kestus@hanmail.net
To the Editor,

Anomalies of and variations in the coronary arteries are important in pathophysiology and the treatment of cardiovascular diseases. The left anterior descending (LAD) coronary artery has the most constant origin, course, and distribution in the human heart; anomalies are rare. Dual LAD was first reported and classified into four types by Spindola-Franco et al. [1] Among these four types, a type 4 anomaly comprises two LADs: a short LAD that originates from the left coronary arteries, terminates in the middle of the anterior interventricular sulcus (AIVS), and does not reach the apex, and a long LAD that originates from the right coronary artery (RCA) transverse to the right ventricular infundibulum, enters the AIVS, and courses to the apex. Type 4 dual LAD is one of the rarest of coronary anomalies. Here, we report a rare case of dual LAD arising from the left and right coronary arteries with superimposed atherosclerotic coronary artery disease (CAD).

A 69-year-old man presented to our cardiology department with chest pain, which was aggravated by fast walking and relieved by rest. His medical history included surgery for spinal stenosis, prolonged steroid use, and iatrogenic Cushing syndrome. He had a 6o-packyear history of smoking, but had quit 3 years earlier. No specific findings were obtained from a physical examination and laboratory testing other than serum hepatitis B virus surface antigen positivity. A normal chest X-ray was obtained. An electrocardiogram showed a normal sinus rhythm without any remarkable abnormalities. Transthoracic echocardiography revealed suspicious hypokinesia at the posterolateral wall with aortic valve sclerosis and an ejection fraction of $57 \%$. The patient also underwent cardiac single-photon emission computed tomography (SPECT), which showed an irreversible photon deficiency in the septum and reversible photon deficiency in the anterior wall and apex.

To evaluate the patient's chest pain and rule out $\mathrm{CAD}$, we performed coronary angiography using the standard right femoral Judkin technique. A left coronary angiogram revealed a short LAD and left circumflex artery (LCX). The LAD was not visualized near the apicoseptal region of the left ventricle. The mid and distal portions of the LAD were avascular and free of collateral circulation. The LAD traveled normally and terminated after the small second septal and diagonal branches (Fig. 1A). A selective right coronary angiogram revealed an anomalous branch that originated from the proximal RCA and coursed very closely with the short LAD, which originated from the left main artery in the AIVS and terminated at the apex (Fig. 1B). We interpreted this angiographic finding as 


\section{KJIM ${ }^{-}$}
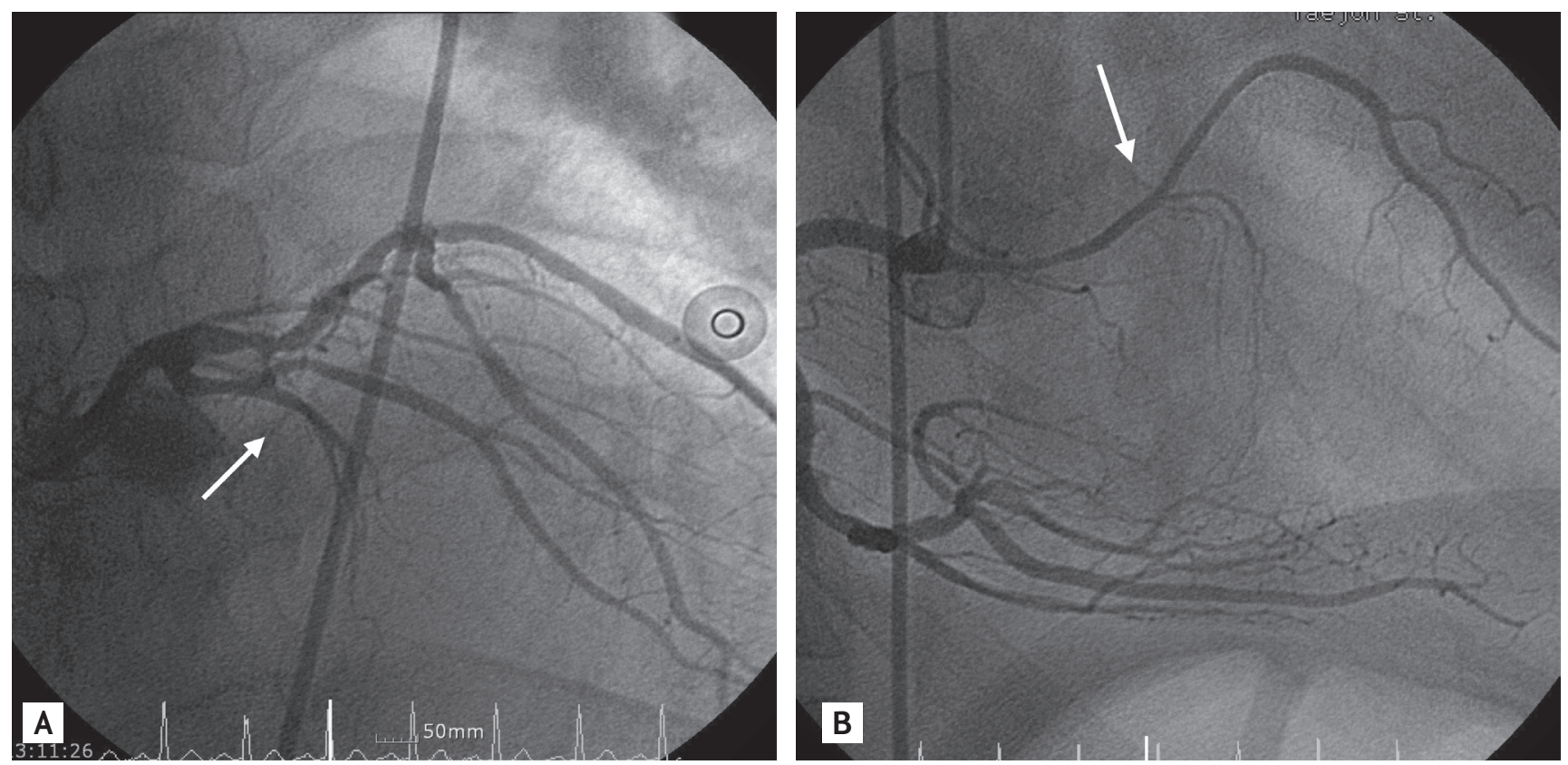

Figure 1. A coronary angiogram showing the left anterior descending (LAD). (A) A short LAD (arrow) was found to terminate in the middle of the anterior interventricular sulcus after small second septal and diagonal branches. (B) An anomalous branch from the proximal right coronary artery (arrow) that terminated at the apex was detected.

the LAD, supplied by both the left main coronary artery and RCA. Significant atherosclerotic stenosis was detected in the proximal and middle portions of the LCX (Fig. 2A). On-site percutaneous coronary intervention (PCI) was performed for the LCX with a $3.5 \times 24.0-\mathrm{mm}$ Pico stent for the proximal lesion and a $3.0 \times 30.0-\mathrm{mm}$ Pico stent for the middle lesion (Fig. 2B). The patient was discharged 1 day after PCI without complications.

The broad application of coronary angiography has revealed many anomalies of the coronary arteries that vary in number, origin, course, distribution, and termination point. In 1983, Spindola-Franco et al. [1] reported 23 cases of dual LAD and classified the anomaly into four subtypes according to the origin and course of the long $\mathrm{LAD}$, as follows.

Type 1: the short LAD runs in the AIVS and is generally the source of all major proximal septal perforators. The long LAD also runs in the AIVS, descending on the left ventricular side of the AIVS and reentering the distal AIVS to reach the apex.

Type 2: the short LAD is the same as in type 1, but the long LAD descends over the right ventricular side before reentering the AIVS.

Type 3: the short LAD is consistent with that in types 1 and 2 . The long LAD travels intramyocardially in the ventricular septum.

Type 4: high in the AIVS, a very short vessel is formed by the LAD proper and the short LAD. The major septal perforators and diagonal branches originate from this vessel. The long LAD is from the RCA.

Type 1 to 3 anomalies arise separately from the proximal part of the LAD and are divided into two left coronary arteries. In a type $4 \mathrm{LAD}$, the LAD is supplied by the LAD proper and the RCA. It is extremely rare among the four types; only a few cases have been reported worldwide to date [1-5]. Kunimoto et al. [2] reported a case of type 4 dual LAD confirmed by both multidetector-row computed tomography and coronary angiography, while Kosar [4] reported a type 4 dual LAD found incidentally in a patient with LCX stenosis. In our patient, the long LAD originated from the RCA and coursed very closely with a short LAD originating from the left main artery in the AIVS that terminated at the apex. Therefore, this anomaly was classified as a type IV dual LAD.

Dual LAD is benign in nature and usually asymptomatic. However, when atherosclerotic CAD is present, it is difficult to differentiate major stenosis or occlusion of the mid or distal portion of the LAD from this anomaly. Whether or not this anomaly can precipitate CAD has 

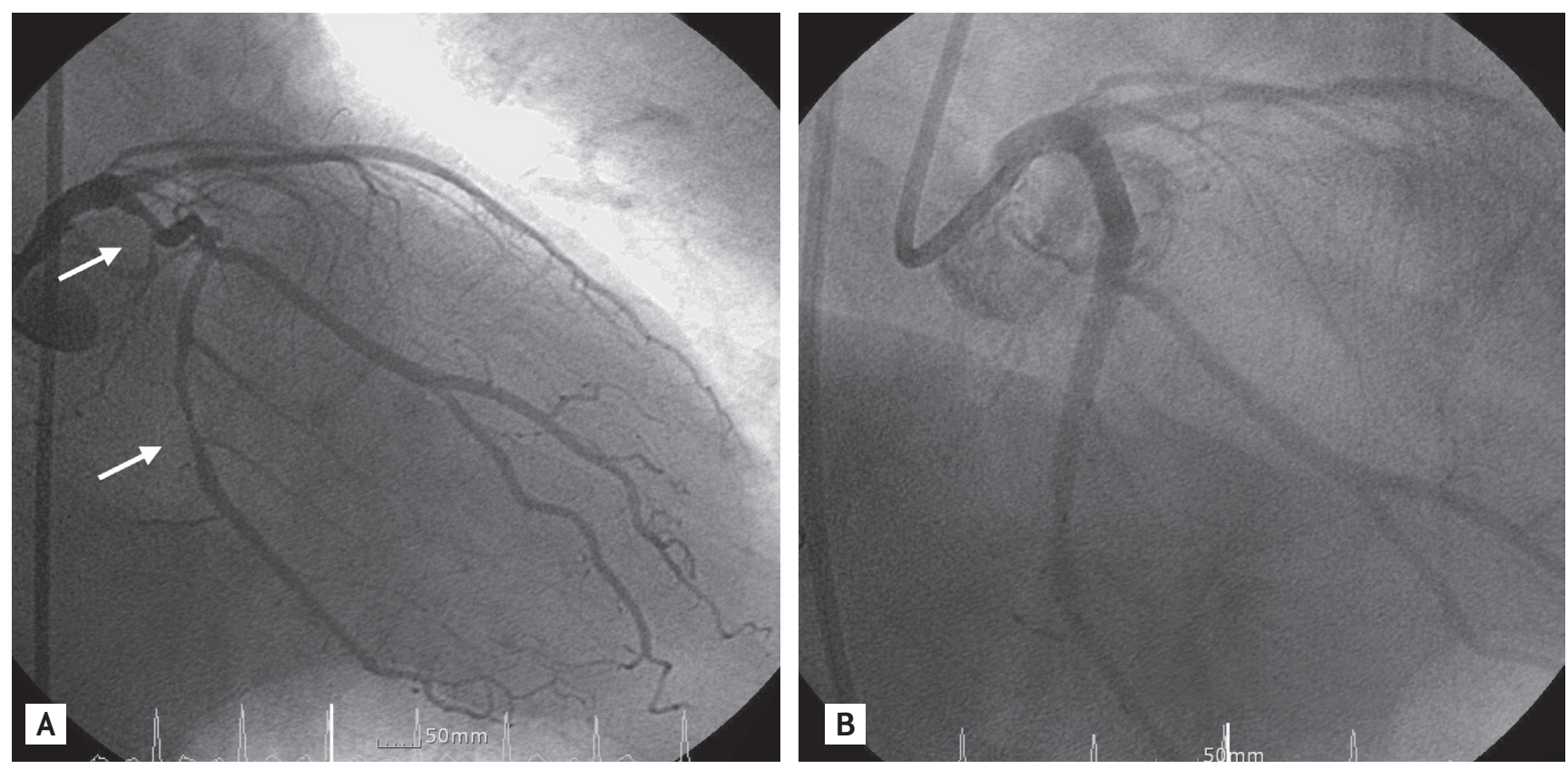

Figure 2. A coronary angiogram showing the left circumflex artery (LCX). (A) Critical stenosis was seen in the proximal and middle LCX (arrows). (B) Successful coronary intervention for the LCX was performed using two Pico stents and balloon angioplasty.

not been established.

The angiographic evaluation of coronary artery anomalies is crucial for both coronary artery intervention and surgery involving the coronary arteries [1-5]. In patients with suspected acute coronary syndrome superimposed by coronary artery anomalies, a mismatch may occur between the results of noninvasive studies of the involved vessel (e.g., echocardiography or cardiac SPECT) and those of coronary angiography. Knowledge of these anomalies would help clinicians locate the correct culprit lesion and prevent erroneous decisions. Spindola-Franco et al. [1] described a patient with type 4 dual LAD and an acute ventricular septal defect in the apical portion of the interventricular septum. Occlusion of the RCA and severe disease of the long LAD explained the apical septal rupture in the presence of a patent LAD proper and a normal, short LAD [1].

In conclusion, we treated a patient with type 4 dual LAD and an atherosclerotic lesion in the LCX. Being alert to this coronary artery anomaly will help clinicians to diagnose and manage patients properly. To our knowledge, this is the first such case in Korea.

Keywords: Coronary artery anomaly; Left anterior descending artery

\section{Conflict of interest}

No potential conflict of interest relevant to this article was reported.

\section{REFERENCES}

1. Spindola-Franco H, Grose R, Solomon N. Dual left anterior descending coronary artery: angiographic description of important variants and surgical implications. Am Heart J 1983;105:445-455.

2. Kunimoto S, Sato Y, Kunimasa T, et al. Double left anterior descending artery arising from the left and right coronary arteries: depiction at multidetector-row computed tomography. Int J Cardiol 2009;132:e54-e56.

3. Voudris V, Salachas A, Saounotsou M, et al. Double left anterior descending artery originating from the left and right coronary artery: a rare coronary artery anomaly. Cathet Cardiovasc Diagn 1993;30:45-47.

4. Kosar F. An unusual case of double anterior descending artery originating from the left and right coronary arteries. Heart Vessels 2006;21:385-387.

5. Yoshikai M, Kamohara K, Fumoto H, Kawasaki H. Dual left anterior descending coronary artery: report of a case. Surg Today 2004;34:453-455. 